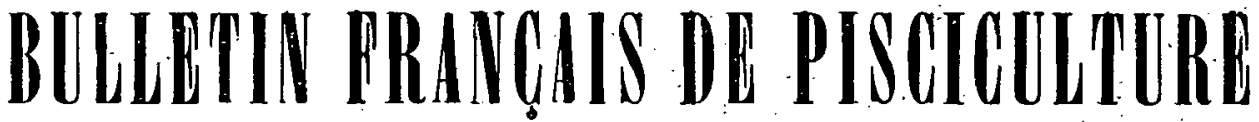

\section{LE GRAND SILURE ET SON ÉLEVAGE PAR LES CARPICULTEURS HONGROIS}

\author{
Par le Docteur EMILE UNGER \\ Chargé de cours à l'Université de Budapest.
}

C'est une réussite inattendue que celle de l'acclimatation, dans 'les étangs à Carpes, d'un monstrueux vorace du bassin du Janube dont le pcids peut atteindre jusqu'à 2 ou même 3 quintaux. Pourtant, elle à été réalisẻe en Hongrie.

Ce poisson $\because$ le grand Silure (Silurus glanis L.), , est peu connü dans l'Ouest de l'Europe, où on ne le rencontre pas. Dans les contrées du Nórd et du Centre, oü il n'est pas rare, il n'est pas très apprécié des consommäteurs. Par exemple, en Prusse èt dans les parties septentrioriales et occidentales de l'Empire allemand, sa valeur commerciale est minime. Les pêcheurs ont, en outre, de fortes préventions contre cet ichthyophage redoutable.

A l'inverse, sur le teritoire arrosé par le Danube, ò̀ le Silure devient de plus en plus commun dans les rivières et lacs à meŝre qu'on s'avancé vers l'Est, il est de mieux en miéux estimé par les pêcheurs et par les clients des poissonneries. En Hongrie, son prix se hausse presque au niveau de celúi des espèces les plus répútées, telles que le Sterlét (A cipeñser ruthenus L.) ou le Sandre (Luciopercä sandra Cuvv); il dépassé toujours celui de la Carpe (Cyprinus carpio,L.) et du Brochet (Esox lucius L.):

Il existe, au regard de celte variabilité dans l'estime dont le Silure est l'objet, une sonte de correspondance avec la fav̀eur, dont bénéficic l'Anguille (Anguilla anguilla L. $_{\text {. }}$ Ce dernier pósson étant très rare dans les régions danubiennes, Je Silure, au point de vue commerciạl; le remplace comme aýant une cháir de qualité similaire, plutôt grrasse: Un vieux et très grand spécimen ne représente certainement pas un mets délicieux, parce que trop lourd. Paí contre, un sujet de 2 à 3 kilogrạmes; de la longueur d'une Angüille, se substitue parfaitement à cette dernière : sa chair est savoureuse et dépourvue d'arêtes. En Hongriè or lui donne même la préférencé, quel que soit l'accommodement culinaire.

Sur le menu de la plupart dés bons hôtels el restaurants de Budapest; 
on peut, presque quotidiennement, voir ligurer le Silure sous son nom magyar de "Harcsa ) (r).

Ce poisson est, au surplus, l'élément fondamental de l' “ Halàszlé », la si renommée soupe aux poissons de Hongrie. Ce potage original et national est préparé; en plein air, par les pềcheurs du Danube et de la Tisza, avec quelques Sterlets, des Carpes en suffisance et une bonnè quantité de Silure.

Naguère, ce poission, de tout temps recherché des gourmets de notre pays, ne se rencontrait qu'en eaux libres. C'est assez récemment que des carpiculteurs magyars se sont avisés d’entreprendre son élevage dans leurs étangs, soit seul, soit associé à d'autres prédateurs, à titre complémentaire.

Adjoindre quelques voraces aux Carpes est un très vieil usage.- En Allemagne, le Brochet esi utilisé pour débarrasser les étangs de tout le fretin, sans valeur au regard de la Carpe, qui y pénètre avec l'eau d'alimentation. Il n'en va pas de mème en Hongrie, le Brochet n'y étant pas fort apprécié et offrant l'inconvénient de s'attaquer aux Carpes pour la satisfaction de son insatiable appétit. Aussi son rôle était-il généralement confié, jusqu'ici, au Brochet-Perche ou Sandre, dont l'élevage est très facile. Beaucoup de carpiculteurs, chaque année, en Avril, mois de la fraye, vendent des œufs fécondés de cette espèce.

Cependant, les essais d'élevage du Sandre en étang n'ont pas, en général, donné grande satisfaction. C'est un poisson peu rustique, ne supportant pas les réductions brusques et amples du taux de l'oxygène dissous, qui surviennent dans les meilleurs étangs de Hongrie. Les Sandres sont les premières et, souvent; les uniques victimes de ces raréfactions. Ils périssent aussi par quantitẻs à l'automne, lor's de la vidarige, dans l'eau trouble et peu profonde.

Quelques autres voraces ont élé essayés et n'ont pas, non plus, répondú à l'altente, tels : la Perche (Perca fluviatilis L.); la Grémille (Acerina cernua L.) ; le Poisson-Chat importé d’Ạmérique (Ameiurus nebulosus Lesuevr) ; et la Perche-Soleil (Eupomotis gibbosus L.). La Perche-Truite ou Black-Bass (Micropterus salmoïdes LACÉPÈDE) ne vaut guère mieux: sa chair est fine, mais, dans les eaux peu profondes des étangs, ce Centrarchide a un accroissement fort lent:

Il est incontestable que le grand Silure est, de tous les carnassiers susceptibles d'être adjoints aux Carpes, celui qui donne, pratiquement, les meilleurs résultats. Il se développe rapidement, se vend à bon prix en

(1) De nos jours, Budapest n'est plus terra incognita dans une région éloignée et barbare du P.oche Orient ; Ia capitale de la Hongrie est réputće pour être la "Reine du Danube ? et " la plus belle cité de l'Europe Centrale ». Les touristes y viennent en foule; je ne saurais trop leur recommander, aụ cours de leur séjour, de goĉter le silure. 
Hongrie, n'a pas de grandes exigences.respiratoires ; au contraire,: mieux que toutes les autres espèces susmentionnées; il s'accommode d'une notable désoxygénation ; dans ces conditions il est; tout commie la ,Carpe; facile à transporter vivant ou à conservẹ tel en réservoirs.

Aussi, dès que l'exploitation des étảngs fut modernisée en Hongrie, plușieurs éleveurs; à même de se procurer de petits Silures dáns les rivières ou lacs de leur voisinage, les introduisirent dans leurs eaux closes.

Mais ce ne fut pas̄ tâchè aisée que d'arriver à utiliser cette espèce de façon courante, comme poisson de comṕlément. Il était nécessaire, pour y parvenir, de disposer, chaque printemps, d'une suffisante abondañcè de jeunes

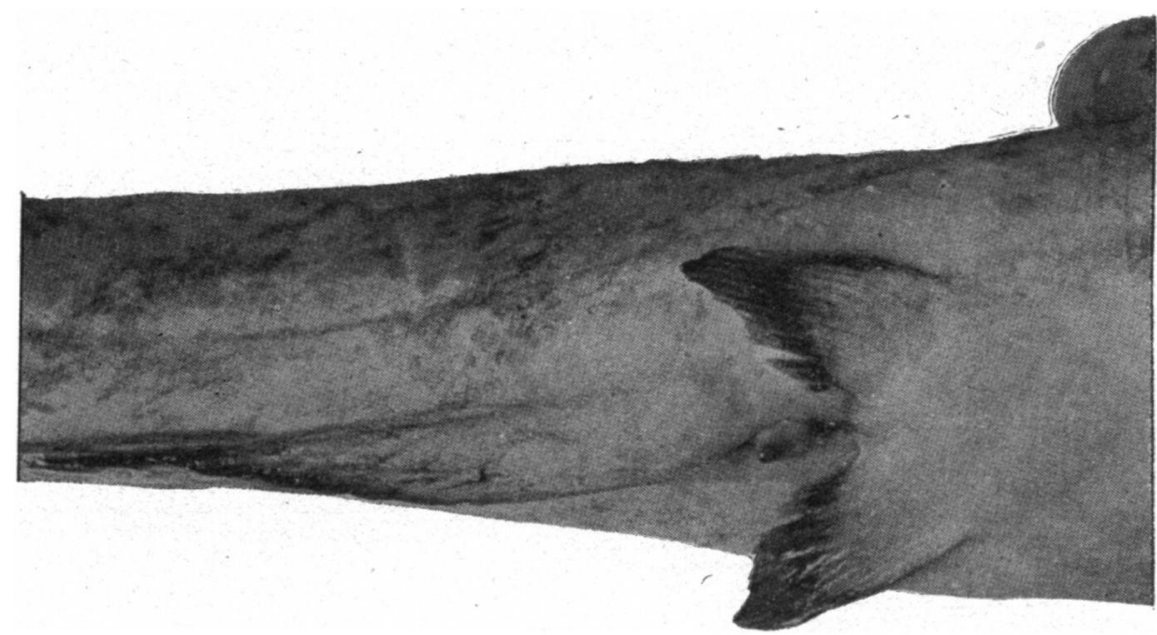

Fıg. 27: - Protúbérance diśtinctive dès sexes chếz le Silure.

Entre les nageoirès abdominales, on voit l'ouverture anale et, immèediatement en arrière, celte protubèrance allongée, perforée à la pöinte, un peu différente chéz le mâle ét.la "femelle. (Cliché UNGer).

presque impossibilité à̀ pêcher, la quạtité requise de petits Silurés sau'vages. On arriva à se procurer des femelles âgées d'au moins quatre ans et pesan 3 kilogrammes ou plus, mais il fut extrêmèment difficile àux carpiculteurs d'avoir à la fois, en temps voülu, mâles et femelles pour réussir la reproduction en eaux closes ; or, ceci étàit nécessaire pour entreprendre ensuite l'élevage des alevins dont ils avaient besoin, soit pour euxmêmes, soit à fins 'commercialès. Au coưrs de ces dernières' années les tentativès couronnées de succès furènt excéptionnelles: D'après M. Zoltan Conchus, qui a, de beaucoup, obtenu les meilleurs résultats; la plupart des échecs sont imputables au fait qu'il est assez rare d'avoir de jeunes mâles parvenus à mạturité sexuélle.

Mais il y a d'autres difficultés. La différenciation des sexes chez lé Silure est très peu accusée, particulièrement s'il s'agit de jeunes géniteurs Les caractères distinctifs sónt ignorés de la plupart de ceux qui - manutentionnent ces poissons. Et on ne trouve rien a ce sujet dans les traités d'ichthyologie óu de pisciculture. 
Cependant le Silure présente, au voisinage immédiat de l'orifice anal, une protubérance, en forme de mamelon. Elle est petite, molle, mais très visible (Fig. 27). Sur un sujet de 75 centimètres de longueur; pesant environ $2 \mathrm{~kg} \mathrm{I} / 2$, cette saillie est longue d'environ $\mathrm{r}_{2}$ millimètres et large, à sa base, de 8 millimètres, approximativement. A l'extrémité se trouve le débouché minuscule de l'urèthre. Or, une certaine différence de forme entre les deux sexes s'accuse pour cette protubérance. Celle du mâle est. plus allongée, plus pointue que celle de la femelle, pour laquelle la configuration est plutôt ovoïde. Mais il faut une certaine habitude pour opérer la distinction.

Une autre cause de maint insuccès dans l'élevage du silure est qu'il est nécessaire d'entretenir un certain couraṇt d'eau dans l'étang durant sa période de fraye, quii, en Hongrie, comprend le mois de Mai et la première quinzaine de Juin.

Mais le plus important, la condition sine qua non de la réussite, est de disposer d'un étang spẹcial, convenable, pour les alevins récemment éclos.

Il ne doit héberger'avec eux, au printemps, aucun autre poisson, car les représentants d'autres espèces font leur proie des jeunes larves du Silure (Fig. 28). Même les Carpillons d'un été óu tous

Fig 28. - Larve vésiculée de Silure récemment éclose : grossie. 5 fois.

poịsons voraces qui sont souvent adjoints à la Carpe dans les étangs. Le Brochet est très précoce ; lors de sa ponte, en Février-Mars, les Carpes ou autres Cyprinides ne s'alimentent pas ou ont un très petit appétit. Le Sandre se reproduit à la fin de Mars ou durant la première moitié du mois d'Avril, rarement plus tard ; ses larves, transparentes comme verre, sont invisibles pour leurs ennemis. Mais celles du Silure éclosent malencontreusement quand les chaleurs printanières se sont fait suffisamment sentir pour dégourdir tous les poissons at les mettre en quête de nourriture.

Il n'est pas nécessaire de nourrir artificiellement les petits Silures pendant les premiers mois de leur existence ; ils trouvent pâture surabondante dans les eaux où ils vivent, du moment où il ne s'y rencontre pas d'autres poissons. Pár ailleurs, ils ne deviennent pas ichthyophages avant la fin de l'été, soit tant que leur. longueur n'est pas au moins de I5 centimètres.

Les petits Silures nouveaux-nés, à -en croire les ouvrages d'ichthyologie, ressembleraient étonnamment à des têtards. Ceci n'est pas tout à fait exact. Nous avons eu l'occasion au printemps dernier d'examiner des alevins porteurs de la vésicule ombilicale, ou venant de la résorber; qui nous ont été obligeamment procurés par M. Concuus ; ils provenaient de 
sa pisciculture justement. renommée d'Ugra, dans le Comitat dẹ Bihar. Les larves en question, soit au moment de leur naissance, soit peu après, offrent une dissemblance avec celles de la Grenouille. Toute personne un peu au courant des questions d'hydrobiologie les reconnaîtra comme n'étant rien autre que de jeunes poissons. La vésicule vitelline est grosse, ovoïde, de couleur jaunâtre, sans globules d'huile. C'est après sa résorption, alors que les organes digestifs se.sont développés, que l'alevin de Silure prend une réelle similitude avec le têtarl, mais seulentent durant unée courte période (Fig: 29). Dès 'qu'il a subi ses premières métamorphoses, il est aisé-

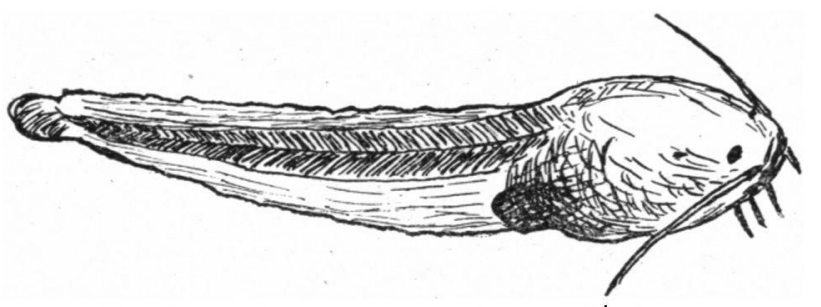

FIg. 29. - Jeune Silure âgé de 15 jours environ.

Grossi 5 fois. (Cloquis UNGEA).

ment reconnaissable aux six barbillons caractéristiques qui s'érigent autour de la bouche $;$ ces appendices ápparáissent de très bonne heure, et même avant disparition complète de la vésicule. Du reste, lèur bourgeon se laisse déjà discerner au microscope chez le poịsson qui vient d'éclore, dont la longueur est seulement de $;$ millimètres.

L'cxamen du contenu stomacal d'une toute jeune larve de ís millimètres, immédiàtément après résorption de la vésicule, nous a fait voir un organe digẹtif bourré de Cyclopes. L'autopsie de deux autres sujets pêchés en Aột, quand ils avaicnt atteint la taille de 1 I centimètres et le poids de 15 grammes (Fig 3o), a montré qu'ils se nourrissaient de minus-

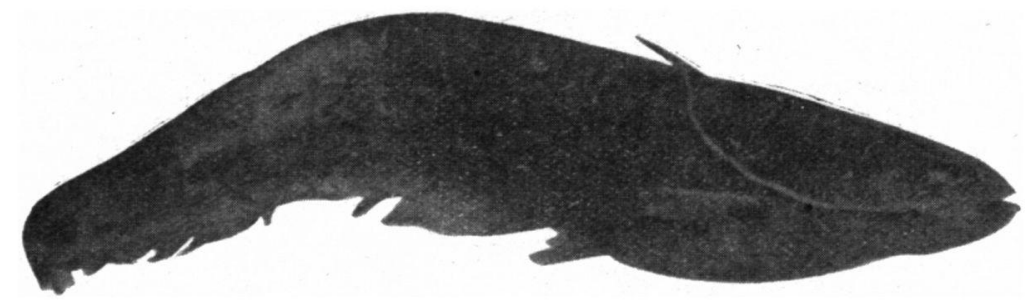

Fıg. 30. - Jeune Silure âgé de 3 mois (Pisciculture de Tata).

Grandeur réelle. (Cliché UNGer).

cules insectes ailés tombés dans l'élang ou ceux, aptères vivant à sa superficie (Collembola).

D'après M. Conchus, lẹ petits Silures, 'encore incapables de s'attaquer aux menus poissons;' 'se repaissent du maïs distribué aux Carpes. Dans șes étangs d'Ugra; il a trouvé plusieurs Silures, au courș dẹ. leur second 
été, qui, pesant environ 250 grammes, avaient l'estomac distendu par le grain dont ils s'étaient gavés.

C'est un fait connu que le Silure se sustente surtout aux dépens de la faune aquatique de fond, mais les grands et vieux sujets s'attaquent également aux. Grenouilles, aux Canards et aux Oies. Ces palmipèdes sont souvent engloutis par eux, de même que les autres oiseaux d'eau. Il n'est donc pas étonnant de voir de jeunes Silures, dans les étangs, explorer la surface, en quête de proies vivantes. Par contre, l'observation de M. Concrnus, qui les fait voir comme accidentellement végétariens; est récllement curieuse.

A la fin de l'été, on peut introduire de petits Cyprinides dans l'étang d'alevinage des Silures pour scrvir d'aliment à ces derniers. Mais cetté intervention est souvent inutile, attendu que dans beaucoup de cas, il y aura suffisante abondance de fretin naturel. Il serait beaucoup plus important d'enlever les géniteurs après la fraye, à la fin de. Juin ou dans les premiers jours de juillet. On sait qu'ils montent la garde auprès des œufs jusqu'à éclosion, ce après quoi, tout d'abord, les parents ne portent aucun préjùdice à leurs petits. Mais quand ceux-ci ont crû jusqu'à une taille où ils peuvent exciter la convoitise de leurs ascendants, mieux vaudrait éliminer ces derniers et les transporter dans. de grands étangs, peuplés seulement de Carpes de 2 ou 3 étés auxquelles ils ne peuvent faire grand mal. Malheureusement cette opération se heurte à de sérieuses difficultés, sinon même à impossibilités.

Au termes de la période de nourrissage des Carpes, qui, en Hongrie, prend fin avec le mois d'Octobre, les petits Silures ont une taille de 20 id 30 centimètres et pèsent de 50 à roo grammes, quelquefois plus et jusqu'à 3 oo grammes (Fig. $3 r$ ). On les conserve, durant la mauvaise saison, en viviers d'hivernage, comme les Carpes, mais il faut les alimenter en leur fournissant, en abondance, soit de menus poissons sauvages, soit de toutes petites feuilles, s'il y en a en excédent.

Au printemps suivant, ces Silures de un été sont utilisés comme poissons de complément dans les étanğs peuplés de Carpes de même âge, à raison de 25 têtes ou plus-par Heclare. Les Silures de deux élés, d'un poids de 800 à r. 800 'grammes, sont souvent trop gros pour être mis avec des Carpés de un été ; ils seraient pour elles des compagnons dangereux. Par conséquent, là où le péuplement comporte des sujets de taille variée, il est indiqué de ne pas leur adjoindre des Silures de plus d'un été. Bien entendu, aucun spécimen de celte espèce ne doit être toléré dans les étangs dè pose.

Les Silures de deux étés peuvent être mis dans les étangs contenạt des Carpes de même âge si ces dernières sont de taille normale. Quand l'empoissonnage est trop petit, il faut le laisser se développer durant plusieurs semaines avant de compléter le peuplement.

Il va de soi que les Silures d'un été peuvent être mêlés sans inconvé- 


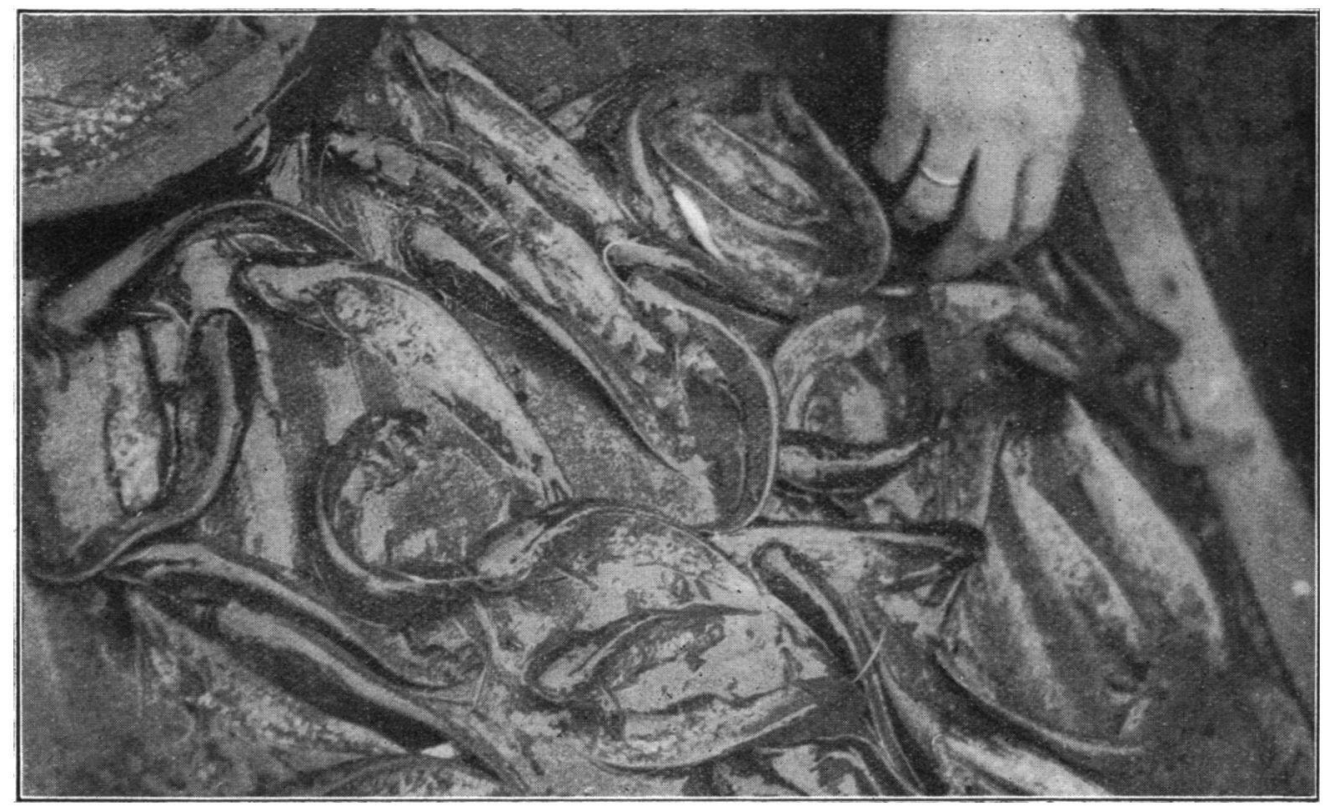

Frg. 31. - Silures d'un été.

Pêchẹ de l'étang d'alevinage à la pisciculture de Tata, appartenant3au Comte F. EsterhazY (17 novembre 1932). (Cliclié ǗvGER).

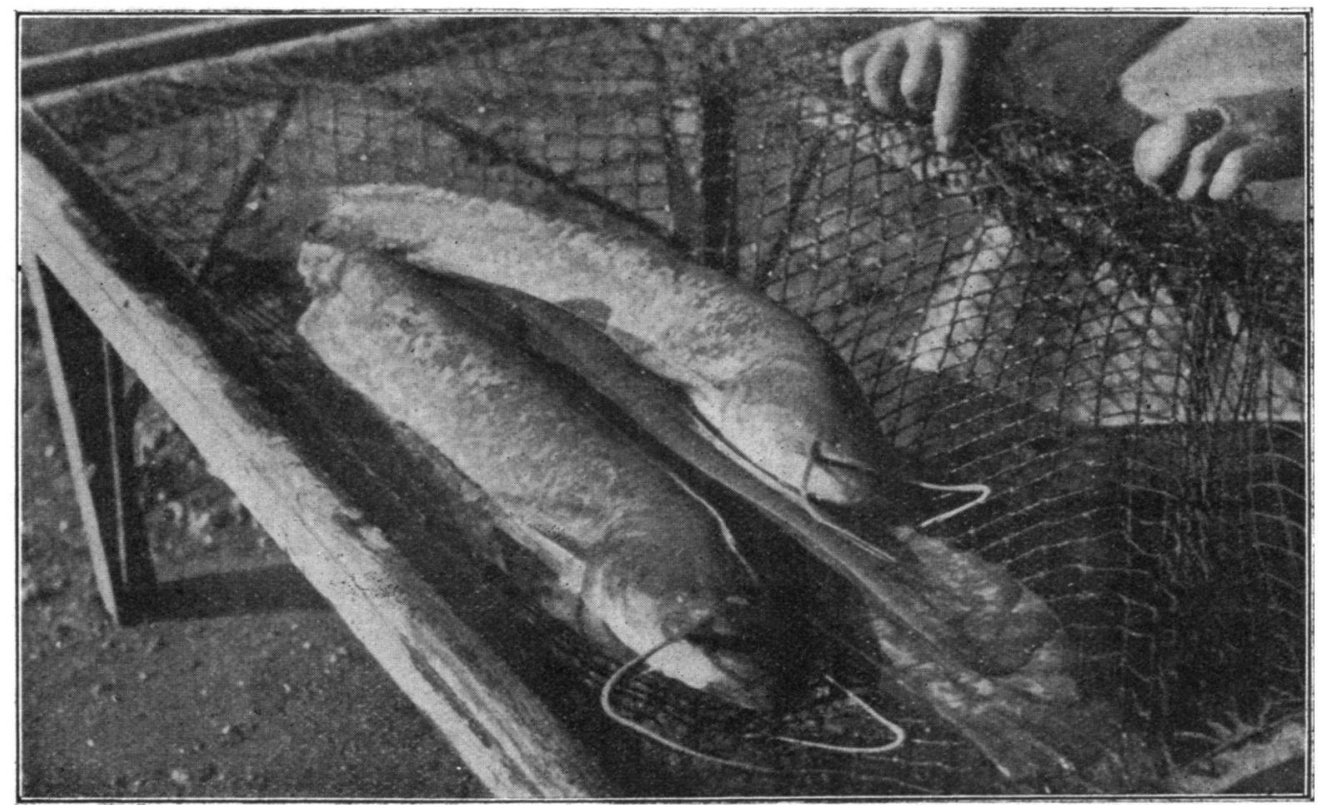

Fug. 32. - Silures de 3 étés.

Pèche du grand étang de la pisciculture de Tata (4 novembre 1932).

(Cliché Uxgen). 
nients à des Carpes plus âgées. On notera qu'un sujet de 2 étés tient, dans un étang à Carpes, le rôle de quatre sujets de I été.

Le Silure de 3 étés atteint au poids de 3 kilogrammes ou davantage (Fig. 32). On le vend à très bon prix avec les plus gros des poissons de 2 étés dont on n'a pas l'emploi. Toutefois, quelques-ums de ces grands exemplaires sont conservés pour servir de géniteurs les années suivantes (Fig. 33).

D'aṕrès les essais faits en Hongrie, les jeunes 'Silures, si l'on prend les précautions requises, ne causent aucuin préjudice aux Carpes et sont très avantageux pour le pisciculteur.

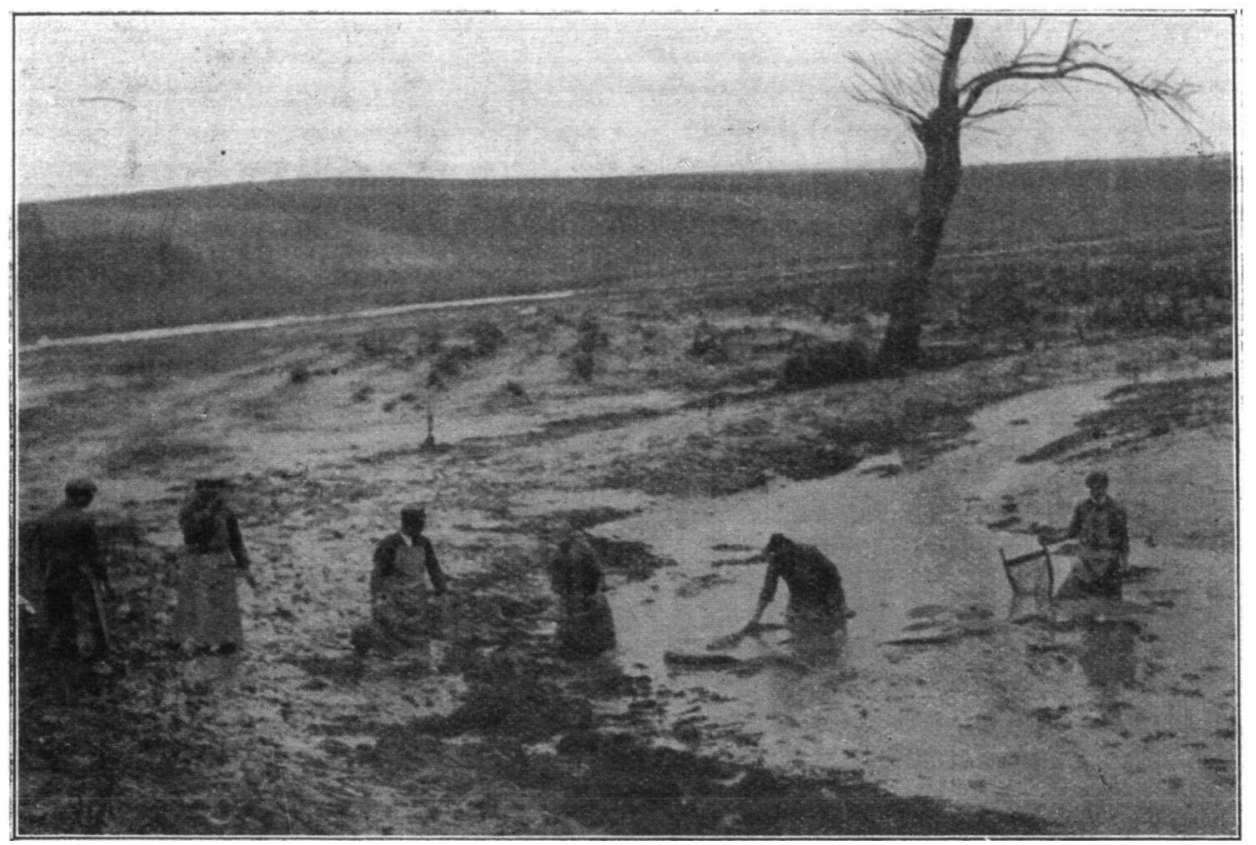

Frg. 33: - Pìche de l'élang d'alevinage des Silures à la pisciculture de Tata (17 novembre 1932). Capture des génitcurs du poids de 10-15 kilogrammes, qu'il eût été préférable d'enlever au coúrs de l'ćté après la fraye.

(Cliché UNGen).

Ils ne détruisent pas seulement les menus poissons sans valeur, Cýprinides ou autres, mais aussi la progéniture des Carpes petites, mais âgées, qui frayent malencontreusement dans les étangs, indésirable progéniture qui fait Je désespoir des carpiculteurs, n'élevant pas des voraces comme poissons de complément.

Enfin, et ce n'est pas là le moindre des services par eux rendus, les Silures ne sont pas du tout exclusifs comme alimentation el font particulièrement la guerre aux Grenouilles et aux têtards, ces hòtes malfaisants des étangs. Ce sont les'meilleurs auxiliaires du pisciculteur pour la lutte contre ces Batracieñs. Aussi les jeunes Silures d'un été en surnombre dans les exploitations où on ne peut ou ne veut poursuivre leur élevage trouvent-ils preneurs ä prix fort rémunérateurs.

On ne s'étonnera pas, qu'un poisson donnant autant de satisfaction que 
le Silure se rencontre maintenant clans la plupart des étangs de Hongrie, entrant dans la composition du peuplement pour une proportion qui varie de I I/2 à 5 pour cent. Cependant, insistons sur ce point quie la fraye et l'ạlevinage ne réussissent pas toujours ni partout. Dé là le prix élevé des sujets de un été $(0,10$ à 0,50 pengö la pièce) .

Comme il a été dit, l'éleveur qui á obtenu le meilleur succès est M. Z. Conchus. A Ugra, dans le Comitat de Bihar, sa production annuelle de Silures âgés dẹ 3-4 étés s'élève à 35 -40 quintaux ; elle ạtteint assez régulièrement la même importancè à Kelabia, dans̄ le Comitat de Bács. Le nombre-des spécimens de I été qui seront dispónibles pour la vente à l'automne prochain est évalué à. 10-12.000.

Toutefois, ce pisciculteur d'avant-garde ne jouit pluș, comme précédemment, d'une sorte de monopole. A Tată à Hortobăgy et àlleứs j̣ est rare; maintenant, que l'alevinage ne réussisse pas ; on obtient donc sur place un empoissonnage dont l'excédent se place facilement avec sérieux bénéfice. En outre, le Comte $F$. Esterhazy est à même de livrer aú commerce après cháque camṕagne, de 30 à 50 quintaux de poíssons de consommation, vieux de 3 ans ou plus.

On peut considérer la technique de l'élevage du grand Silure comme actuellement au point. Peut-êtré les carpiculteurs d'autres pays que la Hongrie trouveraient-ils aussi avantage à introduire dans leurs étangs celte excellente espèce., à tịtre complémentaire.

\title{
Bibliograpuie
}

- I. - RÉpassy Miklós. - Edesvizi halászạt ès halgrázdaság. - Budapest. - $\mathrm{r}^{\mathrm{re}}$ édition, $1908 ;-\mathrm{2}^{\dot{\mathrm{e}}}$ édition, rgi4, p. 203.

2. - B - Gyakorlati tapasztalatók tògazdaságok köréböl. - Halászal, XXIII ; $\div$ 1922, p. 43 .

3. - Bacsá Béla : - A lesö harcsa tenyésztése. - Halászat, XX V̈III ; 1927, p. 81 :

4. - Conchus Zoltán : - Ragadozò halak a tógazdastágban ; -

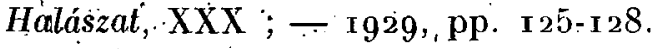

\section{LA MORTALITÉ ESTIVALE DES CARPES SES CAUSES, SES REMÈDES}

\author{
Par M. Miché LHÉRITIER
}

Plsciculteur à Ambazac (Haute-Vienne).

Tous les carpiculteurs des régions lempérées, particulièrement ceux dont les petits étangś ne sont pas ạlimentés par des ruisseaux ou dẹs sourcess, ont constaté qu à certaines époques de l'année, notamment durant la canicule, une quantité souvent considérạble de Cárpẹs mourạit, sans cause 